\title{
Krisendiskurse in der Freien \\ Theaterszene (Berlin): Kunst als soziales \\ Wirksamkeitsversprechen im \\ Spannungsfeld zwischen \\ kulturpolitischer Steuerung und \\ künstlerischer Selbstlegitimierung
}

\section{Anja Quickert}

\section{Zusammenfassung}

In den vergangenen Jahren hat sich die Freie Theaterszene verstärkt mit Fragen der (Selbst-)Legitimierung ihrer künstlerischen Arbeit im Rahmen der strukturellen wie institutionellen Voraussetzungen auseinandergesetzt. Dabei wurde auch der moderne Autonomiebegriff der Kunst nachhaltig infrage gestellt zugunsten einer sozialen Wirksamkeit künstlerischer Praxis. Der folgende Beitrag bildet die diskursiven Positionen dieser künstlerischen Debatte ab, verortet sie in ihrem kunsthistorischen wie gesellschaftlichen Zusammenhang und fragt, welche Funktion die dazugehörigen Krisendiskurse für die (Selbst)Legitimation der Freien Theaterszene im Kontext öffentlicher Kulturförderung besitzen.

\section{A. Quickert ( $\square)$}

Fachbereich II - Germanistik, Universität Trier, Trier, Deutschland

E-Mail: quickert@uni-trier.de 


\section{Schlüsselwörter}

(Selbst-)Legitimation • Freie Theaterszene • Social Turn • Useful Theatre • Kulturpolitische Steuerung

\section{$1 \quad$ Intro: Really Useful Theater?}

„Muss Kunst gesellschaftlich nützlich sein, um einen Wert zu haben? Braucht Theater als Legitimation einen sozialen Auftrag? Oder brauchen wir vielmehr einen Schutz, der Theater und Kunst vor gesellschaftlicher Indienstnahme bewahrt?" Diese kontroverse Fragestellung leitet auf der Homepage der Freien Spielstätte Sophiensaele den Programmtext für das thematische Wochenende „Really Useful Theater" ein. ${ }^{1}$ Unter der kuratorischen Leitung von Stefanie Wenner und Thorsten Eibeler (,,apparatus“) versammelte das mehrtägige, interdisziplinär ausgerichtete Diskurs-Projekt im November 2015 Performances, Vorträge, Diskussionen und Statements von Künstler*innen, Wissenschaftler*innen, Theaterleiter*innen und Kritiker*innen, um die „Debatte um die Freiheit und den Nutzen von Kunst“ auf die Bühne zu bringen, ,das Theater in eine utopische Arena der widerstreitenden Manifeste“ zu verwandeln und die ,teils erbittert geführte Diskussion zwischen den Polen ,Freiheit der Kunst' und ,Nützliche Kunst' " in einem konkreten, gemeinsamen Raum fortzuführen.

Bereits mit dem Titel „Really Useful Theater“ nahm die Veranstaltung ausdrücklich Bezug auf das Konzept einer „Useful Art“, das zu diesem Zeitpunkt bereits im Bereich der bildenden Kunst zu Kontroversen über das grundlegende zeitgenössische Selbstverständnis künstlerischer Praxis geführt hatte. Mit begrifflichen Oppositionen wie „useful theater vs. artistic freedom“, ,participation vs. aestheticism“ umriss die Veranstaltung die Koordinaten des Spannungsfelds, in dem eine kritische Auseinandersetzung der Freien Theaterszene mit Fragen der (Selbst-)Legitimierung künstlerischer Arbeit im Rahmen ihrer strukturellen wie institutionellen Voraussetzungen stattfinden sollte. Der Programmtext benennt dieses Spannungsfeld wie folgt:

\footnotetext{
${ }^{1}$ Das hybride Diskurs-Format ,Really Useful Theater“, gefördert vom Hauptstadtkulturfonds und unterstützt von der Bundeszentrale für Politische Bildung, wurde nach seiner ersten Ausgabe in den Berliner Sophiensaelen 2016 auch im Forum Freies Theater Düsseldorf und auf Kampnagel in Hamburg durchgeführt, jeweils mit Beteiligung der lokalen Freien Theaterszene und Wissenschaftler*innen. Die Autorin dieses Beitrags moderierte gemeinsam mit Veit Sprenger (,Showcase Beat Le Mot“") die erste Abendveranstaltung in Berlin.
} 
„Theater in Deutschland wird subventioniert, die Gesellschaft leistet sich Theater in einem System der Vorfinanzierung, das den Akteuren relative Freiheit in der Umsetzung lässt. Wer aber entscheidet über die Vergabe der Mittel? Nach welchen Kriterien? Ist dieses System, das immerhin schon über 200 Jahre existiert, noch legitim? Müssen Theater auf die aktuelle politische Situation stärker eingehen und sozialarbeiterische Funktionen übernehmen, wie es vermehrt in den Konzepten von Förderinstrumenten formuliert wird? Oder ist das das Ende der Kunst?“ (Sophiensaele 2015).

Auch der Kritiker Janis El-Bira (2015) bescheinigt in seiner Rezension der Veranstaltung auf der Online-Plattform ,nachtkritik“ der Gesellschaft, die ,täglich eine neue, noch nie dagewesene Stufe der Krise oder Bedrohung“ ausrufe, eine zunehmende Erwartungshaltung an die Künstler*ìnnen. „Problemzonen von ,Flüchtlingskrise“ bis ,Terrorwarnstufe 4 “" böten dabei ,eine nicht unwillkommene Legitimierung“, die öffentliche Förderung von Kunst infrage zu stellen: „Welche Relevanz hat das?“

Fragen der „Relevanz“, die Notwendigkeit, die eigene ästhetische Praxis zu diskursivieren und zu legitimieren, sind für die Freie Theaterszene bereits durch ihre institutionellen Rahmenbedingungen existenziell. Indem sie sich wesentlich durch öffentliche Gelder finanziert, die fast ausschließlich für temporäre Projektarbeit vergeben werden, setzt jedes einzelne Projekt eine komplexe Antragsstellung an die jeweiligen Förderinstitutionen voraus. Auf die künstlerische Praxis wirkt sich diese engmaschige Abhängigkeit von Förderstrukturen und Juryentscheidungen stark strukturbildend aus. Einerseits macht sie die Freie Szene für die Steuerung durch kulturpolitische Maßgaben und die Adaption gesellschaftlich dominanter Krisendiskurse besonders anfällig. Andererseits scheint die intrinsische Motivation von Künstler*innen, welche die Prekarität ihrer Arbeitsstrukturen zugunsten ihrer Unabhängigkeit im Produktionsprozess in Kauf nehmen, besonders ausgeprägt zu sein, weshalb Faktoren wie die Selbstlegitimation der künstlerischen Arbeit und die Akzeptanz im Feld einen hohen Stellenwert einnehmen. $^{2}$

Die folgenden Überlegungen wollen den kontroversen zeitgenössischen Krisendiskurs abbilden, der sich um die Forderung nach einer sozialen Wirksamkeit von Kunst im Kontext der Performing Arts herausgebildet hat. Darüber hinaus

\footnotetext{
${ }^{2}$ Die hohe intrinsische Motivation des Feldes wie auch dessen Bereitschaft zur Selbstorganisation und -reflektion lässt sich unschwer an der Vielfalt thematischer Diskussionsveranstaltungen, Weiterbildungsangeboten, Fachtagen und Publikationen, wie sie im Rahmen des Performing Arts Programm in Berlin seit 2013 organisiert werden, nachvollziehen. Darüber hinaus bestätigen die bisherigen Interviews zu Arbeitsbedingungen und Krisendiskursen, die das FOR-Teilprojekt „Markt als Krise“ in Hannover und Berlin mit Akteur*innen der Freien Szene durchgeführt hat, diese Befunde.
} 
versucht der Beitrag, diesen Krisendiskurs durch allgemeine gesellschaftspolitische Entwicklungen zu rahmen und fragt nach den Problematiken, die damit für die Freie Theaterszene hinsichtlich ihres diskursiven Gefüges, ihrer kulturpolitischen Steuerung und ihrer institutionellen Rahmenbedingungen einhergehen.

\section{$2 \quad$ Moderne revisited}

Der Begriff , Useful Art“, der international eine höchst kontrovers geführte Diskussion über die soziale Funktion zeitgenössischer Kunstpraxis ausgelöst hat, geht auf die kubanische Künstlerin Tania Bruguera zurück. Im Jahr 2010 reinstallierte sie eine Kopie des legendären Ready-mades „Fountaine“ von Marcel Duchamps in die Herrentoilette des Queens Museum of Art - ein seriell und maschinell gefertigtes Pissoir, das im Jahr 1917 (nicht nur) die US-amerikanische Kunstwelt über die Frage entzweite, ob ein gewöhnlicher Alltags- und Gebrauchsgegenstand zur Kunst erklärt werden könne (The Blind Man 1917). In unmittelbarer Nachbarschaft des Museums hatte Bruguera zu diesem Zeitpunkt den temporären Hauptsitz ihres Langzeitprojekts „Immigrant Movement International“ eingerichtet - ,a Long-Term art project in the form of an artist initiated socio-political movement" (Bruguera 2010) -, um die Lebensbedingungen von Einwanderern in den öffentlichen Fokus zu rücken. Dabei dürfte es nicht zuletzt der ikonografischen Bedeutung von Duchamps Ready-made für die Kunstgeschichte der Moderne geschuldet sein, dass Brugueras installatives Zitat, verbunden mit ihrem claim nach einer Useful Art - „I have always said that we have to put Duchamp's urinal back in the restroom. Now that urinal is in the restroom of the Queens Museum, you can see it and pee on it.“ (Bruguera 2011) - als paradigmatisches Ereignis im zeitgenössischen Kunstdiskurs wahrgenommen und diskutiert wurde. Die Forderungen nach einer „Useful Art“ stellen dabei die Prämissen der künstlerischen Moderne infrage, wie Florian Malzacher (2015), einer der wichtigsten Kuratoren der deutschsprachigen Freien Szene und einflussreicher Multiplikator einer „Useful Art“, auf der international rezipierten Online-Präsenz des Goethe-Instituts formuliert:

"Of course it is a provocation: after hundreds of years of fighting for the autonomy of art, after decades of learning that the essential quality of art is ambiguity, after years of repeating that art poses rather than answers questions, there is suddenly this persistent call for an art that is useful, for direct commitment, for artistic activism, for intervention in the political reality of our society and economy" (Malzacher 2015). 
Durch diesen „Social“ oder „Ethical turn “ in der Kunst (Bishop 2012), der den Live-Charakter der Performing Arts als Moment tatsächlicher gesellschaftlicher Grenzverhandlung versteht, erfahren nicht nur kollektive Arbeitsprozesse, sondern auch die konzeptuelle Vielfalt an partizipativen, interaktiven oder interventionistischen Formaten eine Aufwertung als ,politische Kunst“ (Malzacher 2020). Entsprechend richtet sich die Selbstlegitimation künstlerischer Arbeit an Kriterien ihrer sozialen Relevanz aus und fordert ethische Aspekte für ihre Bewertung ein. Bis zum Einbruch der Moderne - so der französische Philosoph Jacques Rancière - erschöpfte sich die Funktion der Kunst darin, Teil der Polizei zu sein. Das heißt, sie schrieb herrschende Normen und Machtverhältnisse in ein ästhetisches Regime fort, indem sie die Aufteilung des Sinnlichen (Rancière 2008a) organisierte - in ein Sichtbares und ein Unsichtbares. Kunst repräsentierte etwas, dessen Erscheinen in der Welt durch die herrschende Ordnung legitimiert war und/oder auf dem Normenkanon des Schönen beruhte. Erst der Kunstbegriff der Moderne forderte seit dem 18. Jahrhundert Gleichheit ein - alles kann zum Sujet der Kunst werden - und Gleichgültigkeit - es gibt keine normativen Regeln für ihre Rezeption. Damit begründet Rancière ein Spannungsfeld zwischen Autonomie und Heteronomie, das ein politisches, emanzipatorisches Potenzial beinhaltet: Wer oder was wird sichtbar, wer oder was bleibt unsichtbar? Indem Kunst Körper, Stimmen, Gesten, Objekte in Erscheinung treten lässt, ihnen eine Bühne gibt, legitimiert sie deren Existenz. Dabei setzt dieses emanzipatorische Moment Rancière zufolge allerdings immer einen konstitutiven Dissens voraus, auf den im Folgenden noch einzugehen sein wird.

\section{$3 \quad$ Krisendiskurse und Neoliberalismus}

Den systemischen Zusammenbruch der sozialistischen Staaten 1989, mit dem auch das Ende eines staatspolitisch fundierten Gleichheitsgedankens einherging, haben viele Theoretiker*innen als Moment der Beschleunigung eines vermeintlich alternativlosen Modells des globalen Neoliberalismus gelesen. Die seitdem auch verstärkt diskutierte Krise einer Kunst, die in den veränderten gesellschaftlichen wie wirtschaftlichen Bedingungen um ihre Selbstbegründung und Legitimation ringt, wird von vielen Intellektuellen, Wissenschaftler*innen, aber auch Künstler*innen in direktem Zusammenhang mit diesen globalen Umwälzungen betrachtet (Rancière 2008a; Bishop 2012).

Im Jahr 2008 konstatiert Jacques Rancière eine Diskurslandschaft, die deutlich zeigt, „dass auf dem Feld des Ästhetischen heute ein Kampf ausgetragen wird, der gestern noch den Versprechungen der Emanzipation [...] galt.“ Als 
symptomatisch ,für das zeitgenössische Hin und Her von Ästhetik und Politik" bewertet er dabei „die Entwicklung des situationistischen Diskurses“, der in den 1960er Jahren zur „radikalen Kritik der Politik wurde und heute von der Gewöhnlichkeit jenes Diskurses aufgesogen wird, der die bestehende Ordnung 'kritisch' verdoppelt.“ Nach dem „Ende der politischen Utopien“ entwickelte sich ,die Kunsttheorie zu dem Ort, an dem sich die Dramaturgie des ursprünglichen Abgrunds des Denkens und die Katastrophe seiner Verkennung fortsetzte." (Rancière 2008a, S. 22).

Was Rancière aus kunstgeschichtlicher Perspektive als ,radikale Kritik der Politik" des situationistischen Diskurses bezeichnet, haben Luc Boltanski und Eve Chiapello (2003) aus soziologischer Sicht als Ursache für einen Transformationsprozess identifiziert, den sie als ,Neuen Geist des Kapitalismus' bezeichnen. Infolge der Künstlerkritik unter Legitimationsdruck geraten, adaptiert der Kapitalismus seinerseits seit Ende der 1960er Jahre deren paradigmatische Forderungen und Prämissen: Autonomie, Authentizität, Individualität und Kreativität. Verstärkt durch beispielsweise Richard Floridas Modell sozio-ökonomischer Umwegrentabilitäten einer „Creative Class“, das kreativen Prozessen und diversifizierten Lebensstilen eine neue Legitimität im Kontext von ökonomischen Wertschöpfungsketten der „New Economy“ verschaffte (Florida 2002), verwandelt sich der mobile, kreative und innovative Künstlertypus zum Role Model eines avancierten Neoliberalismus - vor allem aufgrund der für ihn typischen flexiblen, prekären Projektarbeit. Auch wenn sich die Freie Theaterszene wesentlich durch Gelder staatlich subventionierter Förderinstitutionen (und privater Stiftungen) finanziert, operiert sie - der „Creative Class“ vergleichbar - in einem System temporärer Projekt- und Beschäftigungsverhältnisse, in dem die Legitimation ihrer künstlerischen Arbeit durch die Vorgaben staatlicher Förderpolitik an Kriterien wie „Innovation“, „Modellhaftigkeit“, „(inter-)nationale Ausstrahlung“ und

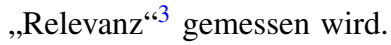

Im Kontext dieses „Förderdispositivs“ (Badura 2014) erscheint es fast folgerichtig, dass sich die Freie Theaterszene von dem absetzt, was allgemein als

\footnotetext{
${ }^{3}$ So lautet beispielsweise eine Frage, die im Rahmen eines Projektantrags an den Hauptstadtkulturfonds in Berlin zu beantworten ist: „Nach dem Hauptstadtfinanzierungsvertrag von 2007 fördert der Hauptstadtkulturfonds Projekte mit gesamtstaatlicher Repräsentation. Dies umfasst Projekte, die für Berlin bedeutsam sind, (inter-)nationale Ausstrahlung haben und besonders innovativ sind. Worin besteht Ihrer Meinung nach die Relevanz bzw. der Modellcharakter Ihres Projekts? An welche aktuellen (inter-)nationalen Diskurse knüpft Ihr Projekt an? Welche innovativen Ansätze werden dabei verfolgt? Welche neuen Perspektiven werden entwickelt?“
} 
neoliberale Prämisse oder „Kreativitätsimperativ“ (Reckwitz 2012) gilt und stattdessen eine ideelle Nähe zu Formen klassischer Sozialkritik sucht. Zumal sich die politische Linke, die Sozialdemokratie und die Gewerkschaften ihrerseits verstärkt seit Ende der 1990er Jahre auf ein konsensfähiges Modell des ,Dritten Weges` (Giddens 2000) zubewegt haben, der die globalisierte Vermarktlichung zwar als regulierbar, dennoch als gesetzt annimmt. Dies hat, folgt man der Politologin und Theoretikerin Chantal Mouffe $(2007,2010)$, dazu geführt, dass sich die westlichen Demokratien in einer postpolitischen Perspektive eingerichtet haben (Mouffe 2007, 2010), in der politisches Handeln sinnlos erscheint, weil es keinen Gegner mehr hat.

Auch das Freie Theater, das sich in Deutschland ursprünglich Ende der 1960er Jahre explizit als kritische Reaktion auf und als (weniger entfremdete, egalitäre, politisch und gesellschaftlich relevante) Alternative zu der hierarchisch und arbeitsteilig organisierten Institution Stadttheater ausgebildet hatte (Fülle 2016), steht mittlerweile nicht mehr nur unter Legitimationsdruck seitens der kulturpolitischen Förderinstitutionen, sondern auch unter Selbstlegitimationsdruck.

\section{$4 \quad$ Repolitisierung und Identitätspolitik der Freien Theaterszene}

Infolge der sich spätestens seit der 2012 erschienenen Studie „Kulturinfakt“ häufenden Krisendiskurse und Legitimitätsdebatten um die institutionalisierte deutsche Theaterlandschaft, die vielerorts eher als kulturelles Erbe Deutschlands ${ }^{4}$ verstanden wird denn als künstlerische Produktionsstätte und die immense öffentliche Gelder zur Erhaltung ihrer Infrastruktur benötigt, wird die Freie Theaterszene gerne als kostensparendes Innovationsmodell in Opposition gebracht. Angesichts der Beharrungskraft anachronistisch anmutender institutioneller Strukturen im Stadttheater, denen eine fortschreitende Heterogenisierung und Diversifizierung der Gesellschaft gegenübersteht, wird die Freie Szene entweder aus kulturpolitischer Sicht instrumentalisiert (Fülle 2016), aus Perspektive eines rationalisierten Kulturmanagements zur ökonomischen und demokratischen Alternative stilisiert (Schmidt 2017, 2019) oder gleich als neoliberales Feindbild identifiziert (Ostermeier 2013). Dabei interessiert sich der vorherrschende öffentliche Diskurs kaum für die Krisendiskurse, die innerhalb der Freien Theaterszene vorherrschen. Da diese - anders als die etablierten, zumeist historischen und unter Denkmalschutz

\footnotetext{
${ }^{4}$ Vgl. die Diskussion um den Antrag des Deutschen Bühnenvereins im Jahr 2018 an die UNESCO zur Aufnahme der Theaterlandschaft als Weltkulturerbe.
} 
stehenden Gebäudekomplexe der Repertoirebetriebe - ihre Existenz und Subventionierung gemäß der institutionalisierten Förderlogik permanent neu begründen muss, sind diese Diskurse unmittelbar mit der künstlerischen wie sozialen Positionierung und (Selbst-)Legitimierung verbunden. Daher reflektieren die aktuellen Krisengefüge der Szene ein paradoxales Spannungsfeld zwischen (individueller) Professionalisierung, die unter neoliberalen Vorzeichen stattfindet, dem Wunsch nach Institutionalisierung, um dem prekären, neoliberalen Dispositiv zu entgehen, dem Versuch einer Repolitisierung, um diese Institutionalisierung als sozial und gesellschaftlich relevanter Akteur zu legitimieren, und der künstlerischen Autonomie, die ihrerseits im Widerspruch zur angestrebten sozialen gesellschaftlichen Funktion und deren Legitimation steht.

Wirft man einen Blick auf die jüngsten Entwicklungen in der deutschen Theaterlandschaft allgemein, so fällt auf, dass sich seit 2011 viele neue Initiativen gegründet und in Vereinen institutionalisiert haben, die klassische Positionen linker Sozialkritik besetzen. Im Sinne einer emanzipatorischen linken Identitätspolitik begründen sie ihre Existenz mit den Defiziten gesellschaftlicher Gleichheit und Gleichstellung im institutionellen Feld Theater: darunter „Bühnenwatch“ (Critical Whiteness), „Pro Quote Bühne“ (Feminismus), ,art but fair“ und „ensemble-netzwerk“ (soziale Arbeitsbedingungen) sowie zuletzt „Die Vielen“" (Verbund gegen rechte politische Strömungen). ${ }^{5}$ Diese Institutionalisierung von Kritik lässt Rückschlüsse auf einen grundlegenden Krisendiskurs der bestehenden Institutionen zu. Doch obwohl die Freie Theaterszene im Vergleich zur Stadt- und Staatstheaterlandschaft ausgesprochen egalitär und divers ausgeprägt ist $^{6}$ - gerade auch hinsichtlich der Merkmale Gender oder People of Color - lässt sich an ihr der Einfluss des ,social turn“ am stärksten beobachten und abbilden. Das spiegeln nicht nur Programmatiken und Ästhetiken der Freien Szene wieder - sie scheint sich selbst zum Impulsgeber und Change Agent für die gesamte Theaterlandschaft entwickeln zu wollen.

Bereits ein oberflächlicher Blick auf die Festivals der zwei wichtigsten Berliner Spielstätten - das HAU Hebbel am Ufer und die Sophiensaele - in den letzten anderthalb Spielzeiten zeigt eine erstaunliche Akkumulation thematischer Schwerpunktsetzungen. Dabei luden die Festivals einerseits individuell produzierte (internationale) Gastspiele aus anderen Städten ein und rahmten sie neu. Sie

\footnotetext{
${ }^{5}$ Erwähnenswert ist hier, dass sich der Verein „Die Vielen“, der fast die gesamte Kulturinstitutionslandschaft zu einer gemeinsamen Solidaritätsbekundung gegen rechte Einflussnahme bewegt hat, aus der Freien Szene heraus initiiert wurde, dem Feld mit den geringsten Ressourcen.

${ }^{6}$ Das hat auch die statistische Auswertung des Theatertreffens 2019 ergeben, die im Rahmen der Konferenz „Burning Issues“ veröffentlicht wurde.
} 
initiierten aber ebenso durch festivaleigene Produktionsmittel eine Vielzahl neuer künstlerischer Produktionen, die passgenau zu der inhaltlichen Setzung der Festivals erarbeitet wurden. Thematisch suchten die Festivals sowohl den Anschluss an translokale gesellschaftliche Diskurse wie postkoloniale Perspektiven (,After Europe“), Feminismus („The Future is Female“), queere Geschlechtsidentitäten (,The Present Is Not Enough. Performing Queer Histories and Futures“), Inklusion („No Limits, Akteure mit Behinderung“) oder Therapie (,Save your soul“), sie fokussierten aber auch Krisendiskurse, die zwar translokal anschlussfähig sind, aber in Berlin eine besondere Relevanz besitzen, wie der Ost/West-Konflikt (,Das Ost/West Ding, DDR neu erzählen!") oder die Stadtentwicklungs- und Gentrifi-

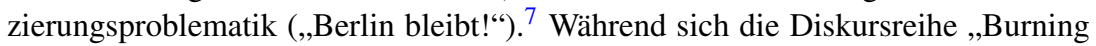
Futures“ im HAU Hebbel am Ufer in der Spielzeit 2019/2020 mit „Ecologies of Existence“ auseinandersetzt, beschäftigen sich die Sophiensaele bereits seit 2018 in der Reihe „Politics of Love“ mit Fragen der Repräsentation in Theater und Gesellschaft.

\section{$5 \quad$ Agenda Setting}

Dem Grund für diese augenscheinliche Verdichtung der identitätspolitischen Agenden einerseits und dem Aufgreifen gesellschaftlicher Krisendiskurse andererseits, kann man sich mit der Idee des von Bernard C. Cohen (1963) entwickelten Agenda Settings annähern. Demnach besitzen Medien allgemein - und das Theater wahrscheinlich insbesondere - zwar keinen unmittelbar nachweisbaren Einfluss auf die Meinungsbildung ihres Publikums. Trotzdem präfigurieren sie die Themen und Phänomene, über die überhaupt nachgedacht werden kann, weil sie im öffentlichen Diskurs vorkommen und Aufmerksamkeit erhalten: Sie präfigurieren also die Wahrnehmung von Wirklichkeit. Wenn es einem Medium gelingt, eine bestimmte Agenda erfolgreich zu etablieren, beispielsweise durch Kumulationseffekte, wirkt deren Relevanz auf den Sender der Botschaft zurück - der im Falle des Theaters gleichzeitig eine Institution ist. Das aus einer Vielzahl solcher Rückkopplungseffekte entstehende Agenda Setting etabliert auf diese Weise ein spezifisches Profil, das sich als Legitimation manifestiert, wenn erfolgreiche Beziehungswirkungen entstehen. Indem das Theater Medium und Institution

\footnotetext{
${ }^{7}$ Auch das Spielstätten-unabhängige (Anti-)Wagner-Musiktheater-Festival „Berlin is not Bayreuth“, initiiert vom Kollektiv ,glanz\&krawall“, das sich zuvorderst als künstlerisches Statement lesen lässt, nahm implizit auf den Stadt- und Gentrifizierungsdiskurs Bezug.
} 
gleichzeitig ist, kann und muss es diese Beziehungswirkungen gleich auf mehreren Ebenen verhandeln: Mit dem tatsächlich teilnehmenden Publikum und mit der Theaterkritik als medialer Verstärkung öffentlicher Relevanz. Beide Faktoren beeinflussen die Wechselwirkung der Institution mit der sie fördernden Kulturpolitik, die ihrerseits eine Legitimation für ihre öffentlichen Ausgaben benötigt.

\section{Paradoxale Wirkungen des „Social Turn“}

Diversifizierung und Repräsentation diverser gesellschaftlicher Gruppierungen sind ohne deren Unterscheidung und Zuordnung nach identitätspolitischen Merkmalen (Geschlecht, Klasse, Migrationshintergrund, körperliche Behinderung) nicht möglich. Paradoxerweise ist diese merkmalsbezogene Unterscheidung gleichzeitig der Grund ihres vorgängigen Ausschlusses aus gesellschaftlichen Bereichen, die zumeist mit einem höheren sozialen Status und größerer Gestaltungsmacht einhergehen. Diese spezifische Paradoxie, das „Double Bind“, eröffnet auch für die Freie Theaterszene diverse Problemfelder, die sich bis in die zeitgenössischen Bühnenästhetiken und -formate abbilden.

Eine grundlegende Problematik ergibt sich bereits aus der asymmetrischen Konstellation (vor allem bei partizipativen Projekten), in der eine zwar prekäre, aber dennoch privilegierte, weil gut ausgebildete und sozial integrierte Schicht die Künstler*innen - Geld und sozialen Status damit erwerben, dass sie weniger privilegierte oder sogar stigmatisierte Randgruppen öffentlich in Erscheinung treten lassen und dabei auch über die Repräsentation dieser Personen entscheiden. Als künstlerische Verfahrensweise, die Merkmale sozialer Ungleichheit über die Machtverhältnisse des künstlerischen Prozesses in die Inszenierung fortschreibt, gilt diese Praxis mittlerweile in weiten Teilen der Freien Szene als delegitimiert ${ }^{8}$. Ein weiteres, sich für die Akteure der Freien Theaterszene auch ganz pragmatisch stellendes Problem der fortschreitenden Diversitäts- und Identitätsanforderungen an die künstlerische Praxis besteht darin, dass die eigenen Identitätsmerkmale und Erfahrungshorizonte über die Legitimität einer Inszenierung entscheiden. Tendenziell entsteht auch dadurch ein normatives Feld, das identitäre Merkmale naturalisiert und die künstlerische Praxis weitgehend mit der Biografie von Künstler*innen gleichsetzt. Damit verliert der Raum der Kunst - sowohl im konkreten

\footnotetext{
${ }^{8}$ Dies kann man aus zahlreichen Podiumsdebatten, Publikationen aus migrantischer Perspektive sowie den bereits erwähnten qualitativen Interviews mit Akteur*innen der Freien Szene schließen.
} 
als auch übertragenen Sinne - zunehmend seine doppelte Bestimmung, zugleich ein sozialer als auch ein fiktionaler, Konsequenz verminderter Raum zu sein. Seine Ambiguität, eine der zentralen Wirkungsweisen moderner ästhetischer Kunsterfahrung, wird durch eine eindeutig lesbare, ethisch fundierte Haltung ersetzt - ein Problem, das auch Rancière (2008b) beschreibt. Ihm zufolge tendiert eine von ihm ,ethische Kunst“ genannte Praxis dazu, sowohl das Ästhetische als auch das Politische zu eliminieren, weil ihr Regime notwendiger Weise konsensuell verfährt und das Konsensuelle keinesfalls widerständig oder politisch ist (Rancière 2008b).

Die Gefahr einer konkreten (kultur-)politischen Vereinnahmung der Künste problematisiert auch die US-amerikanische Kunstphilosophin Claire Bishop (2012) in ihrem Buch „Artificial Hells: Participatory Art and the Politics of Spectatorship“, in dem sie die enge Verflechtung des künstlerischen „Social Turns“ mit der gesellschaftlich-politischen Entwicklung von New Labour ab 1997 in Großbritannien herausarbeitet. Darin führt sie plausibel aus, wie der Terminus „Social Exclusion“ zur „Key Phrase“ einer kulturpolitischen Periode avanciert, die eine neue gesellschaftliche Opposition schuf - nämlich die „Included Majority“ und die „Excluded Minority“. Demnach erhielt nach dem Eintritt von New Labour in die Regierung der Gedanke neue Konjunktur, dass die staatliche Förderung von Kunst legitimiert sei, wenn/weil die Künste sozial inklusiv wirkten, was im Übrigen auch eine unüberschaubare Flut an Forschungsliteratur und Studien zeigte, um künstlerische Praxis als Universallösung für gesellschaftliche Probleme und Krisen zu etablieren. Dabei arbeitet Bishop unter Berufung auf verschiedene Soziologen, beispielsweise Beck und Beck-Gernsheim (2002), heraus, dass die Ursachen für die diversen „Social Exclusions“ fast ausschließlich im Individuellen und Biografischen der exkludierten Personen(-gruppen) gesucht werden - Familienprobleme, Drogen, Kriminalität usw. - die strukturellen Ausschlussmechanismen im politischen, sozialen und ökonomischen Kontext dagegen vernachlässigt würden. So betrachtet, kann das Versprechen von insbesondere partizipativen, inklusiven Projekten, seine Beteiligten zu „empowern“, auch dahin gehend interpretiert werden, dass neoliberale Staatsgefüge vermittels der Künste auch marginalisierte soziale Existenzen zu mehr Selbstverantwortung mobilisieren möchten - und dergestalt systemische gesellschaftliche Disparitäten und Verwerfungen verschleiern. Folgt man diesen kritischen Betrachtungen, bietet ein „Social Turn“, der dem Wunsch folgt, sich über soziale Funktionen stärker gesellschaftlich und selbst zu legitimieren (und zu institutionalisieren), um sich vom temporären, neoliberalen Arbeits- und Institutionsmodell zu distanzieren, keine grundsätzliche Alternative zum Problem der allgemeinen Vermarktlichung. Zudem erscheint gerade im Bereich der Freien Szene die Steuerungsmöglichkeit 
durch die Kulturpolitik problematisch, insofern diese über gezielte Förderrichtlinien und programmatische Vorgaben künstlerische Projekte als Äquivalenz sozialpolitischer Regulierung vereinnahmen können.

\section{$7 \quad$ Schluss}

Die Forderung nach gesellschaftlicher Relevanz, nach einer künstlerischen Praxis, die ein soziales Wirksamkeitsversprechen beinhaltet, stellt seitens der Förderinstitutionen ein standardisiertes Kriterium zur Bewilligung öffentlicher Zuwendungen für freie Künstler*innen dar und dient der Legitimation öffentlicher Förderung. Darüber hinaus ist in den letzten Jahren - nicht zuletzt aus dem Feld der Freien Szene selber und unter Einfluss von internationalen Diskursen der bildenden Kunst - die Forderung nach einer „Useful Art“, nach einem „Social Turn“ als (Selbst-)Legitimation und Sinnstiftung künstlerischer Praxis laut geworden. Dieser künstlerische (Krisen-)Diskurs reagiert auf den Abbau nationalstaatlicher Sozialpolitik, gewerkschaftlicher Vertretung durch die Heterogenisierung von Arbeitsprozessen sowie das Ende der sozialen Utopien nach 1989. Indem die Freie Szene Theater als sozialen Raum betrachtet, der auch in seinen institutionellen Strukturen und Produktionsprozessen eine sozial gerechte und diverse gesellschaftliche Teilhabe einlösen soll, setzt sie über ihre gesellschaftspolitisch adressierten Krisendiskurse Trends für die gesamte deutsche Theaterlandschaft. Auf die künstlerische Praxis selbst angewandt, hat die Konjunktur von partizipativen Projekten, Artivismus oder therapeutischen Formaten die Freie Szene allerdings auch programmatisch polarisiert, was öffentliche Formate zu ihrer Selbstverständigung - wie beispielsweise die eingangs beschriebene interdisziplinäre Veranstaltung „Really Useful Theatre“ - belegen. Denn Kunst, verstanden als soziale Praxis, stellt sowohl die ästhetische Dimension einzelner Inszenierungen als auch den modernen Autonomiebegriff des Feldes infrage. Mithin kollidieren damit zwei unterschiedliche Auffassungen von gesellschaftlicher ,Nützlichkeit'- die, wie der Programmtext zu „Really Useful Theatre“ vermerkt, allerdings „hierzulande grundsätzlich außer Zweifel“ steht, Publikationen wie dem „Kulturinfakt“ zum Trotz. Grundsätzlich fraglich ist dennoch, ob sich die ,,Nützlichkeit“ von Kunst nach ihrer unmittelbaren sozialen oder ethischen Wirksamkeit bemessen lässt ${ }^{9}$ oder als abstrakte „Instanz der gesamtgesellschaftlichen Selbstbeobachtung“, wie es der Soziologe Andreas Reckwitz formuliert (Reckwitz 2012, S. 132), wofür

\footnotetext{
${ }^{9}$ Worüber während der Veranstaltung ein großer Dissens zwischen den Akteur*innen bestand.
} 
ihre prinzipielle Ambiguität, ihre „Nutzlosigkeit“, eine wesentliche Voraussetzung ist. Eine in ihrem Selbstverständnis reflexive Freie Szene muss und wird die Frage nach ihrer sozialen Wirksamkeit in einem Krisengefüge zwischen kulturpolitischer Steuerung und künstlerischer Selbstlegitimierung daher fortlaufend verhandeln und sie wird damit weitere Impulse für Transformationsdynamiken des Theaters insgesamt setzen.

\section{Literatur}

Badura, Jens. 2014. Ungerechtfertigt, aber JA. Ein Plädoyer. Konzeptarbeit. https://konzeptar beit.wordpress.com/texte-2/. Zugegriffen: 14. Febr. 2020.

Beck, U. und E. Beck-Gernsheim. 2002. Individualization: Institutionalized Individualism and its Social and Political Consequences. London: Sage.

Bishop, Claire. 2012. Artificial Hells. Participatory Art and the Politics of Spectatorship. London: Verso.

Boltanski, L., und È. Chiapello. 2003. Der neue Geist des Kapitalismus. Konstanz: UVK Verlagsgesellschaft.

Bruguera, Tania. 2010. Immigrant Movement International, Tania Bruguera. https://www. taniabruguera.com/cms/4860Immigrant+Movement+International.htm. Zugegriffen: 12. Jan. 2020.

Bruguera, Tania. 2011. Introduction to Useful Art, Tania Bruguera. https://taniabruguera.com/ cms//528-0-Introduction+on+Useful+Art.htm. Zugegriffen: 13. Febr. 2020.

El-Bira, J. 2015. Really Useful Theater - Die Konferenz zu Sinn und Zweck des Theaters an den Berliner Sophiensaelen als Kraftakt mit großer Qualität. Nachtkritik.https://www.nachtkritik.de/index.php?option=com_content\&view=art icle \&id=11807:really-useful-theater\&catid=53\&Itemid=83. Zugegriffen: 13. Febr. 2020.

Florida, Richard. 2002. The Rise of the Creative Class. New York: Basic.

Fülle, Henning. 2016: Freies Theater. Die Modernisierung der deutschen Theaterlandschaft (1960 - 2010). Berlin: Theater der Zeit.

Giddens, Anthony. 2000. Der Dritte Weg. Die Erneuerung der sozialen Demokratie. Frankfurt: Suhrkamp.

Malzacher, Florian. 2015. Useful Art. What role can art play in politics? Goethe-Institut. https://www.goethe.de/en/kul/tut/gen/tup/20559574.html. Zugegriffen: 18 Jan. 2020.

Malzacher, Florian. 2020. Gesellschaftsspiele. Politisches Theater heute. Berlin: Alexander.

Mouffe, Chantal. 2007. Über das Politische. Wider die kosmopolitische Illusion. Frankfurt: Suhrkamp.

Mouffe, Chantal. 2010. Postdemokratie" und die zunehmende Entpolitisierung. Bundeszentrale für Politische Bildung. https://www.bpb.de/apuz/33565/postdemokratie-und-die-zun ehmende-entpolitisierung-essay?p=all. Zugegriffen: 13. Febr. 2020.

Ostermeier, T. 2013. Ein paar Narren im Dienst der Gesellschaft. Nachtkritik. https://nac htkritik.de/index.php?option=com_content $\&$ view=article $\&$ id=8327:thomas-ostermeierueber-die-zukunft-des-theaters\&catid=101: debatte\&Itemid=84. Zugegriffen: 13. Febr. 2020. 
Reckwitz, Andreas. 2012. Die Erfindung der Kreativität. Zum Prozess gesellschaftlicher Ästhetisierung. Frankfurt: Suhrkamp.

Rancière, Jacques. 2008a. Die Aufteilung des Sinnlichen. Die Politik der Kunst und ihre Paradoxien. Berlin: b_books.

Rancière, Jacques. 2008b. Ist Kunst widerständig? Berlin: Merve Verlag.

Schmidt, Thomas. 2017. Theater, Krise und Reform. Eine Kritik des deutschen Theatersystems. Wiesbaden: Springer.

Schmidt, Thomas. 2019. Macht und Struktur im Theater: Asymmetrien der Macht. Wiesbaden: Springer.

Sophiensaele. 2015. Really Useful Theater, https://sophiensaele.com/de/archiv/festival/rea lly-useful-theater.

The Blind Man. 1917. Tout-faits. The Marcel Duchamps Studies Online Journal. https://www. toutfait.com/issues/issue_3/Collections/girst/Blindman2/5.html. Zugegriffen: 20. Febr. 2020 .

Open Access Dieses Kapitel wird unter der Creative Commons Namensnennung 4.0 International Lizenz (http://creativecommons.org/licenses/by/4.0/deed.de) veröffentlicht, welche die Nutzung, Vervielfältigung, Bearbeitung, Verbreitung und Wiedergabe in jeglichem Medium und Format erlaubt, sofern Sie den/die ursprünglichen Autor(en) und die Quelle ordnungsgemäß nennen, einen Link zur Creative Commons Lizenz beifügen und angeben, ob Änderungen vorgenommen wurden.

Die in diesem Kapitel enthaltenen Bilder und sonstiges Drittmaterial unterliegen ebenfalls der genannten Creative Commons Lizenz, sofern sich aus der Abbildungslegende nichts anderes ergibt. Sofern das betreffende Material nicht unter der genannten Creative Commons Lizenz steht und die betreffende Handlung nicht nach gesetzlichen Vorschriften erlaubt ist, ist für die oben aufgeführten Weiterverwendungen des Materials die Einwilligung des jeweiligen Rechteinhabers einzuholen. 